Original Article (short paper)

\title{
Anxiety increases the blood pressure response during exercise
}

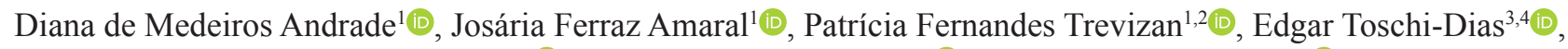 \\ Lilian Pinto da Silva ${ }^{1}$, Mateus Camaroti Laterza ${ }^{1}\left[\begin{array}{c}0 \\ \text {, Daniel Godoy Martinez }\end{array}\right.$ \\ ${ }^{1}$ Universidade Federal de Juiz de Fora, Unidade de Investigação Cardiovascular e Fisiologia do Exercício, Juiz de \\ Fora, MG, Brasil, ${ }^{2}$ Centro Universitário Estácio Juiz de Fora, Juiz de Fora, MG, Brasil, ${ }^{3}$ Universidade Metodista \\ de São Paulo (UMESP), São Paulo, SP, Brasil; ${ }^{4}$ Universidade Ibirapuera (UNIB), São Paulo, SP, Brasil
}

\begin{abstract}
Aim: The hypothesis that higher levels of anxiety promote greater blood pressure (BP) responses during physical exercise is tested. The hypothesis that metaborreflex response is increased in an anxious individual is tested as well. Methods: There were 43 volunteers divided by anxiety level: 12 volunteers with mild, moderate and severe symptoms (anxious-group) and 31 volunteers with a minimum level of anxiety (control-group). Arterial BP, heart rate, and forearm blood flow were measured simultaneously during handgrip exercise and post-exercise ischemia, and forearm vascular resistance (FVR) was calculated. Results: Anxious group present higher systolic, diastolic and mean $\mathrm{BP}$ at rest when compared with control group $(130 \pm 11$ vs. $122 \pm 12 \mathrm{mmHg}, \mathrm{p}=0.048 ; 70 \pm 6$ vs. $65 \pm 8 \mathrm{mmHg}, \mathrm{p}=0.033$; $90 \pm 7$ vs. $84 \pm 9 \mathrm{mmHg}, \mathrm{p}=0.033$, respectively) and higher response of systolic, diastolic and mean BP and FVR during exercise when compared with control group $(20 \pm 9$ vs. $13 \pm 7 \mathrm{mmHg}, \mathrm{p}=0.009 ; 17 \pm 8$ vs. $11 \pm 6 \mathrm{mmHg}, \mathrm{p}=0.006 ; 18 \pm 8$ vs. $11 \pm 6 \mathrm{mmHg}, \mathrm{p}=0.005$; and $0 \pm 13$ vs. $-7 \pm 9$ units, $\mathrm{p}=0,003$, respectively). During post-exercise ischemia, the anxious group also present higher response of diastolic BP, mean BP and FVR when compared with a control group (11 \pm 12 vs. $3 \pm 4 \mathrm{mmHg}, p=0,001,10 \pm 8$ vs. $3 \pm 5 \mathrm{mmHg}, \mathrm{p}=0,002 ; 9 \pm 11$ vs. $-2 \pm 8$ units, $p=0,03$, respectively). Conclusion: Anxious individuals present higher BP responses during physical exercise when compared with those with minimal anxiety symptoms. This increased response may be explained, in part, by increased peripheral vascular resistance due to the greater metaborreflex response.
\end{abstract}

Keywords: anxiety, exercise, blood pressure, vascular resistance.

\section{Introduction}

Anxiety, a transient and normal emotional state throughout life, promotes feelings of discontent, discomfort, or worry about an event with an uncertain outcome ${ }^{1}$. However, when the anxiety level is disproportionate to the stimulus and the anxiety symptoms become chronic, this emotional state may become pathological and can negatively interfere with daily activities ${ }^{1}$.

The Diagnostic and Statistical Manual $5^{2}$ defines generalized anxiety disorder as characterized by "chronic persistent worry" for "at least 6 months" along with at least three of the following six symptoms: restlessness, feeling of being keyed up or 'on edge'; fatigue; difficulty concentrating; irritability; muscle tension; and sleep disturbance ${ }^{2}$.

However there is a condition called anticipatory anxiety, that is a psychological and physiological state associated with the anticipation of threatening stimuli ${ }^{3}$. Under experimental conditions, Lipman, Grossman, Bridges, Hamner, Taylor ${ }^{4}$ suggested an effect of anticipatory anxiety on hemodynamic variables such as mean blood pressure and heart rate, that increase early in the preparation phase and throughout the execution of two mental stress tests (math and speech), as a way of preparing the body for coping or running away, also known as "fight or flight"4.

Currently, physical activity is shown to be associated with decreased symptoms of anxiety in some studies ${ }^{5,6}$. For example, study with middle-aged women, those who had participated in a resistance exercise program presented better mental health as evidenced by lower levels of depression and anxiety compared to sedentary counterpartners ${ }^{7}$. On the other hand, exercise causes important hemodynamic adaptations to ensure body homeostasis under dynamic conditions ${ }^{8}$. During exercise, it is known that greater the information coming from central (central cortex) and peripheral (mechanoreceptors and metaboreceptors) areas, greater will be the stimuli for the cardiovascular control areas located in the central nervous system. Proportionally to stimuli, as an efferent response, there is an increase in adrenergic activity, which in turn increases heart rate, blood pressure, and cardiac output and decreases blood flow in the visceral region ${ }^{8,9,10,11}$. Even though all of these hemodynamic adjustments occur simultaneously to favor increased blood flow to the skeletal muscles during exercise, it has been reported in the literature that exacerbated increases for these hemodynamic responses are associated with a high risk of adverse events on the cardiovascular system ${ }^{12,13,14}$. 
Considering the evidence that anxiety symptoms and the practice of physical exercise promote hemodynamic changes, to date, the effects of the anxiety level on acute hemodynamic response to physical exercise in healthy individuals remain unknown. Thus, the aim of the present study was to test the hypothesis that the higher the anxiety levels promote the higher the blood pressure values at rest and the greater the blood pressure response to exercise. In addition, the hypothesis that the metaboreflex sensitivity is increased in anxious individuals was tested.

\section{Methodology}

\section{Sample}

The sample consisted of 43 volunteers divided into two groups according to the level of anxiety proposed by Beck, Epstein, Brown, Steer ${ }^{15}$. Individuals with mild, moderate, and severe anxiety levels were allocated to the anxiety group (AG) $(n=12)$, while those with minimal anxiety were allocated to the control group (CG) $(n=31)$. The following inclusion criteria were adopted: age between 18 and 50 years, systolic and diastolic blood pressure lower than $140 \mathrm{mmHg}$ and $90 \mathrm{mmHg}$ at rest, respectively, and non-performance of systematized physical exercise for at least three months. Volunteers with cardiometabolic diseases, smokers, individuals taking medications that could affect the cardiovascular and nervous systems or individuals with any musculoskeletal impairment that might impair the performance of the exercise protocol were excluded from the study.

The evaluations were always conducted in the morning. The volunteers were instructed not to ingest alcohol and/or caffeine and not to perform strenuous physical activities in the 24 hours preceding the evaluations.

\section{Ethics Procedure}

All study participants were informed about the research and the procedures to which they would be subjected. In addition, all participants signed the informed consent form as stated by the Brazilian Council of Ethics in research with human subject (CONEP/BR). The present study includes data from two studies approved by the Research Ethics Committee $\left(\mathrm{n}^{\circ} .720 .370\right.$ and 374/2011) in accordance with the norms of the 1996 Helsinki Convention and National Health Council Resolution 466/2012.

\section{Anxiety Level Assessment}

The anxiety level was assessed using the Beck Anxiety Inventory $(\mathrm{BAI})^{15}$. The questionnaire evaluates the level of anxiety in the last seven days and was applied on the same day as the experimental protocol, under the supervision of an experienced psychologist (ETD).

The $\mathrm{BAI}^{15}$ is a self-report instrument containing a list of 21 questions addressing common anxiety symptoms answered on a four-point ascending scale corresponding to the level of anxiety. The anxiety level was classified according to the total score into the categories minimal (0-7), mild (8-15), moderate (16-25), and severe (26-63) anxiety ${ }^{15}$.

\section{Anthropometric Assessments}

For weight and height measurements, a Líder ${ }^{\circledR}$ scale (Araçatuba, SP, Brazil) with 0.1-kg precision and a graded stadiometer with $0.5-\mathrm{cm}$ precision coupled to the scale were used. At the time of evaluation, the volunteers wore light clothing and were barefoot. The variables weight and height were measured according to the criteria described by the American College of Sports Medicine ${ }^{16}$. The body mass index was calculated by dividing the weight by height squared $\left(\mathrm{kg} / \mathrm{m}^{2}\right)$.

\section{Blood Pressure and Heart Rate}

During rest and exercise, blood pressure was automatically measured minute-by-minute in the right leg using the oscillometric method via a Dixtal ${ }^{\circledR}$ model 2023 multiparameter monitor (Manaus, AM, Brazil) ${ }^{17}$.

The heart rate was continuously recorded throughout the protocol using 5 skin electrodes placed according to the standard lead provided by the 5-way cable of the same Dixtal ${ }^{\circledR}$ model 2023 multiparameter monitor (Manaus, AM, Brazil) ${ }^{17}$.

\section{Forearm Blood Flow}

Forearm blood flow was evaluated using venous occlusion plethysmography (Hokanson ${ }^{\circledR}$ Plethysmograph, Washington, DC, USA). The volunteer was placed in a supine position, and his non-dominant arm was raised above the heart level to ensure adequate venous drainage. A mercuryfilled silastic tube connected to the low-pressure transducer and a plethysmograph was placed around his non-dominant forearm $5 \mathrm{~cm}$ from humeroradial joint ${ }^{17}$.

A cuff was placed around his wrist and another around the top of the volunteer's arm. The cuff placed around his wrist $(7 \times 20 \mathrm{~cm})$ was inflated to a supra-systolic pressure (200 $\mathrm{mmHg}) 1$ minute before starting the measurements and was kept inflated throughout the procedure. At 15 -second intervals, the cuff placed around his arm $(12 \times 23 \mathrm{~cm})$ was inflated to a supravenous pressure $(60 \mathrm{mmHg})$ for a period of 10 seconds and was then deflated quickly and kept deflated for the same period. This procedure was repeated 4 times per minute ${ }^{18}$. The increased tension in the silastic tube reflected 
the increased forearm volume and, consequently, indirectly, the increased muscle blood flow in the forearm, evaluated in $\mathrm{ml} / \mathrm{min} / 100 \mathrm{ml}$. The forearm muscle blood flow waveform was acquired in real-time via computer using NIVP3 software (Washington, DC, USA) ${ }^{18}$.

The local peripheral (forearm) vascular resistance was calculated by dividing the mean blood pressure by the forearm blood flow and was reported in units.

\section{Handgrip Exercise Experimental Protocol}

The isometric handgrip exercise was performed using a Jamar ${ }^{\circledR}$ dynamometer (Tupã, SP, Brazil). For this purpose, first, the maximum isometric handgrip strength was calculated by taking the mean of 3 maximum voluntary contraction attempts performed with the dominant limb.

The protocol consisted of 3 minutes of rest, during which the volunteer remained in a supine position in a quiet and air-conditioned room. Next, 3 minutes of physical exercise at $30 \%$ of maximum voluntary contraction was performed to determine the magnitude of the hemodynamic responses during the activation of the central command and muscle mechanoreceptors and metaboreceptors. Afterward, for the isolated evaluation of muscle metaboreflex, a cuff placed around the arm was inflated to supra-systolic pressure (200 $\mathrm{mmHg}$ ) to promote circulatory occlusion for 2 minutes. Therefore, the physical exercise protocol consisted of 3 minutes of rest, 3 minutes of isometric exercise at $30 \%$ of maximal voluntary contraction, and 2 minutes of circulatory occlusion, characterizing the post-exercise ischemia ${ }^{17}$.

\section{Statistical Analysis}

For evaluation of the descriptive characteristics of the sample, the mean and standard deviation of the mean were used. ShapiroWilk test was used to test the normality of the distribution of all variables.

The differences related to the characteristics of the control and anxious groups were tested using Student's t-test for independent samples for the variables that presented normal distribution and the Mann-Whitney for the variables that violated this assumption. The t-test was preceded by Levene's test for equality of variances. The chi-square test was used to test differences in the sex ratio between the groups. Two-way analysis of variance for repeated measures followed by Fisher's post hoc test was used to test differences between the cardiovascular responses of the groups during the proposed exercise.

The correlations between the anxiety level and blood pressure both at rest and during exercise were tested using the Spearman correlation test.

The statistical significance level adopted was $p \leq 0.05$. All statistical analyses were performed using IBM SPSS ${ }^{\circledR} 20$ for Windows (Chicago, IL, USA).

\section{Results}

The anxiety scores and physical characteristics of both groups are described in Table 1. As expected, the anxious group had a higher anxiety score than the control group. Individuals in the anxious and control groups had similar physical characteristics.

Table 1. Anxiety Scores and Physical Characteristics.

\begin{tabular}{lccc}
\hline \multicolumn{1}{c}{ Variable } & Anxious Group & Control Group & P value \\
\hline Anxiety score & $18 \pm 11$ & $3 \pm 2$ & 0.001 \\
Sex $(\mathrm{M} / \mathrm{F})$ & $2 / 10$ & $9 / 22$ & 0.405 \\
Age $($ years $)$ & $34 \pm 7$ & $31 \pm 8$ & 0.259 \\
Weight $(\mathrm{kg})$ & $67.7 \pm 13.6$ & $67.6 \pm 14.6$ & 0.850 \\
Height $(\mathrm{m})$ & $1.62 \pm 0.06$ & $1.66 \pm 0.09$ & 0.147 \\
BMI $\left(\mathrm{kg} / \mathrm{m}^{2}\right)$ & $25.6 \pm 4.6$ & $23.9 \pm 3.3$ & 0.316
\end{tabular}

Values are the means \pm SDs. BMI, body mass index.

The hemodynamic characteristics at rest of the volunteers are presented in Table 2. The systolic, diastolic, and resting mean blood pressures were higher in the anxious group than in the control group $(130 \pm 11$ vs. $122 \pm 12 \mathrm{mmHg}, \mathrm{p}=0.048$; $70 \pm 6$ vs. $65 \pm 8 \mathrm{mmHg}, \mathrm{p}=0.033$; and $90 \pm 7$ vs. $64 \pm 8 \mathrm{mmHg}$, $\mathrm{p}=0.033$, respectively), while the heart rate, muscle blood flow, and resting forearm vascular resistance were similar for the two groups.

Table 2. Hemodynamic Characteristics at Rest.

\begin{tabular}{lccc}
\hline \multicolumn{1}{c}{ Variable } & Anxious Group & Control Group & P value \\
\hline SBP (mmHg) & $130 \pm 11$ & $122 \pm 12$ & 0.048 \\
DBP (mmHg) & $70 \pm 6$ & $65 \pm 8$ & 0.033 \\
MBP (mmHg) & $90 \pm 7$ & $84 \pm 9$ & 0.033 \\
HR (bpm) & $67 \pm 10$ & $64 \pm 8$ & 0.380 \\
FBF (ml/min/100 ml) & $2.2 \pm 0.7$ & $2.3 \pm 0.7$ & 0.741 \\
FVR (units) & $43.8 \pm 13.8$ & $40.4 \pm 12.7$ & 0.445 \\
\hline
\end{tabular}

Values are the means \pm SDs. SBP, systolic blood pressure; DBP, diastolic blood pressure; MBP, mean blood pressure; HR, heart rate; $\mathrm{MBF}$, forearm blood flow; FVR, forearm vascular resistance.

The SBP, DBP, MBP, and heart rate patterns during the exercise protocol are shown in Figure 1 (panel A,B,C and $\mathrm{D}$ respectively). Pressure and heart rate increases were observed in both groups compared with the resting condition. However, in the anxious group, the pressure increase was more exacerbated when compared with the control group. In contrast, the heart rate increased significantly and similarly in both groups (Figure 1; panel D).

During exercise, the increased muscle blood flow response was similar for the two groups (Figure 2; panel A). However, when we corrected the muscle blood flow according to the mean blood pressure, we observed a decrease in the forearm vascular resistance during exercise in the control group and an increase in this variable in the anxious group. In addition, the forearm vascular resistance values of the anxious group were significantly higher compared with the control group (Figure 2; panel B). 


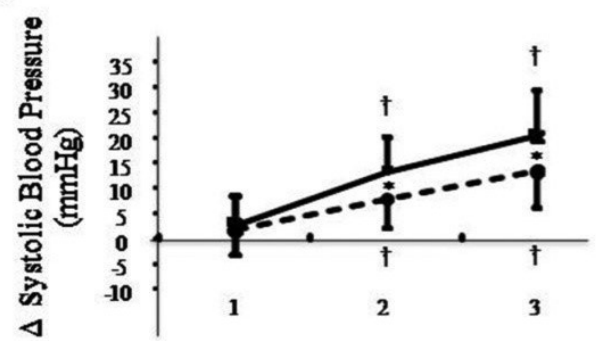

Moderate Exercise (30\% MVC) Time (minutes)

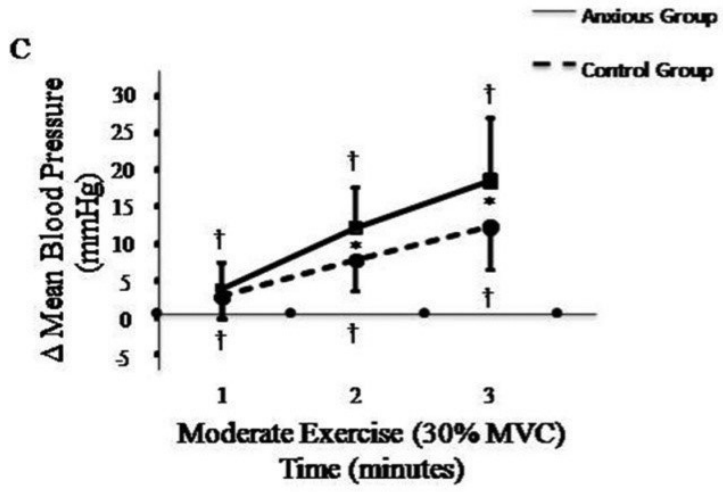

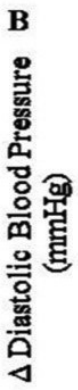

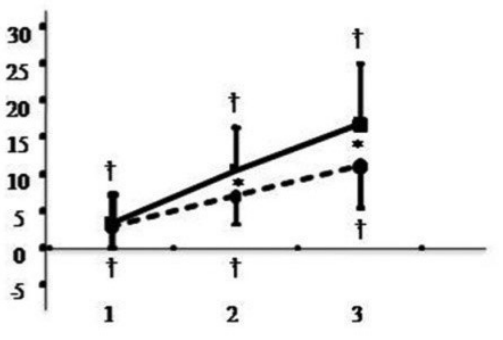

Moderate Exercise (30\% MVC) Time (minutes)

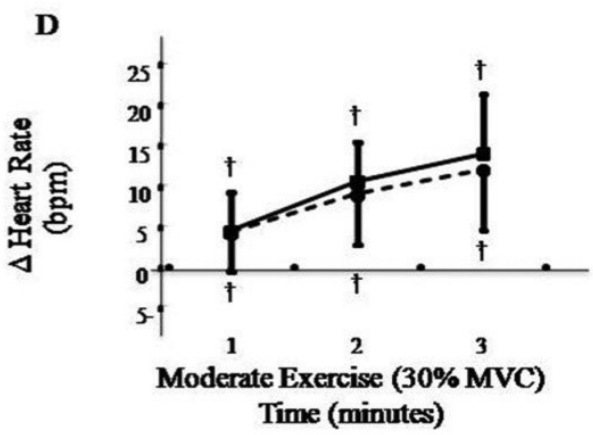

Figure 1. Absolute delta values of the systolic, diastolic, and mean blood pressure ( $\mathrm{mmHg}$ ) and heart rate (bpm) responses during moderate exercise at $30 \%$ of maximal voluntary contraction (panels A, B, C, and D, respectively). Note that there were increases in systolic, diastolic, and mean blood pressure and heart rate levels in both groups when compared with the resting condition during moderate exercise, but the pressure elevation levels were higher in the anxious group than in the control group (panels A, B, and C, respectively). The heart rate increased similarly in both groups. $\mathrm{f} \leq 0.05$ vs. baseline; ${ }^{*} \mathrm{p} \leq 0.05$ vs. group.

$\mathbf{A}$

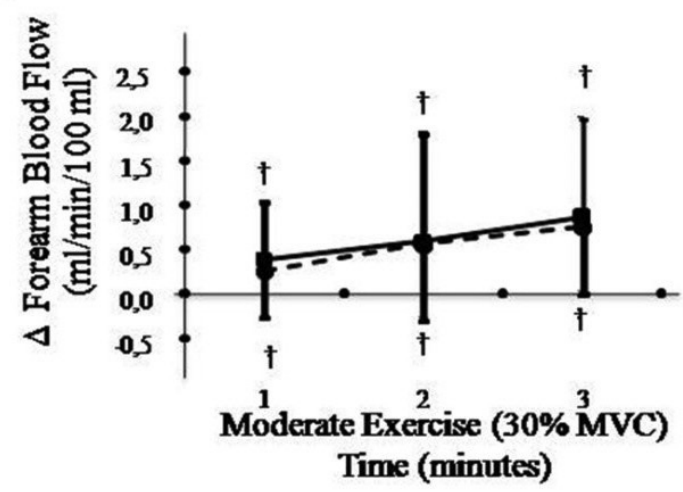

B

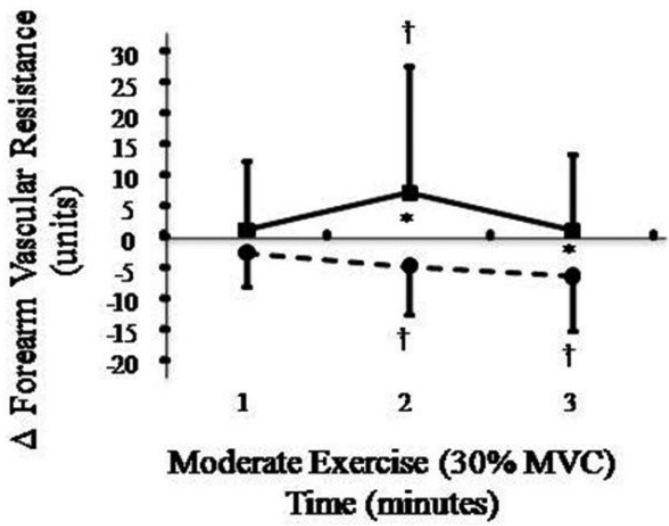

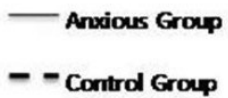

Figure 2. Absolute delta values of the forearm blood flow $(\mathrm{ml} / \mathrm{min} / 100 \mathrm{ml})$ and forearm vascular resistance (units) responses during the exercise protocol at $30 \%$ of maximal voluntary contraction (panels A and B, respectively). Note that muscle blood flow increased significantly and similarly in both groups (panel A); however, forearm vascular resistance decreased in the control group and increased in the anxious group, becoming greater in the anxious group (panel B). Ip $\leq 0.05$ vs. baseline; ${ }^{*} \mathrm{p} \leq 0.05$ vs. group.

In the post-exercise ischemia period for assessment of metaboreflex sensitivity, the systolic, diastolic, and mean blood pressures and heart rate presented higher values with concerning to the resting period in both groups, demonstrating the sympathoexcitatory effect of this maneuvers (Figure 3; panel
A, B, C and D respectively). Despite, the diastolic and mean blood pressure responses were more pronounced in the anxious group than in the control group. The systolic blood pressure and heart rate responses were similar for the two groups (Figure 3; panel A and D respectively). 

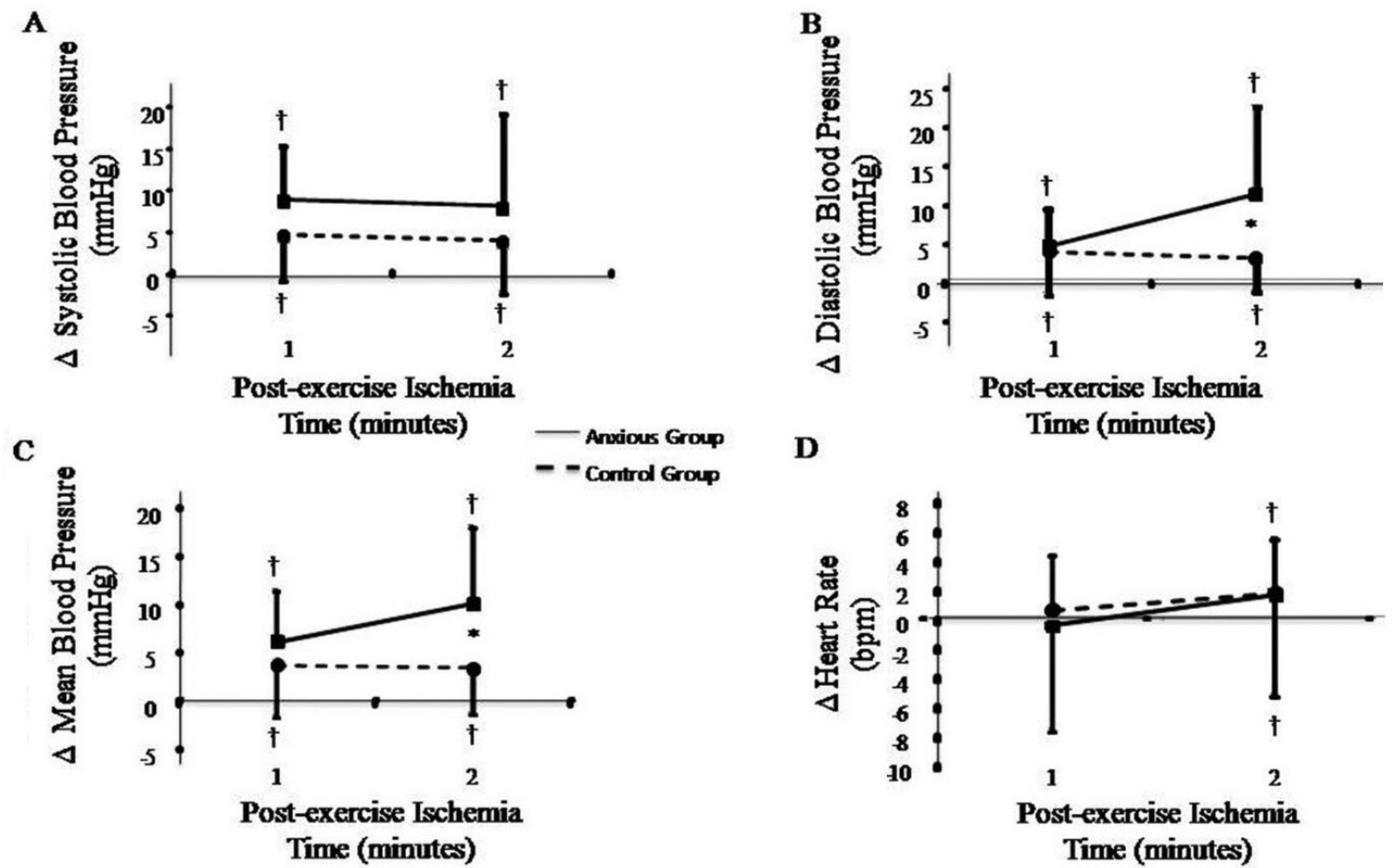

Figure 3. Absolute delta values of the systolic, diastolic, and mean blood pressure (mmHg) and heart rate (bpm) responses during the postexercise ischemia protocol (panels A, B, C, and D, respectively). Note the higher systolic, diastolic, and mean blood pressure and heart rate levels in both groups when compared with the resting condition; however, in the anxious group, the diastolic and mean blood pressures presented higher increases compared with the control group. $\mathrm{f} \mathrm{p} \leq 0.05$ vs. baseline; ${ }^{*} \mathrm{p} \leq 0.05$ vs. group.

Still, during the period of post-exercise ischemia, the muscle blood flow response was similar for the two groups. However, the forearm vascular resistance response was different (Figure 4; panel B) while the control group presented maintenance, the

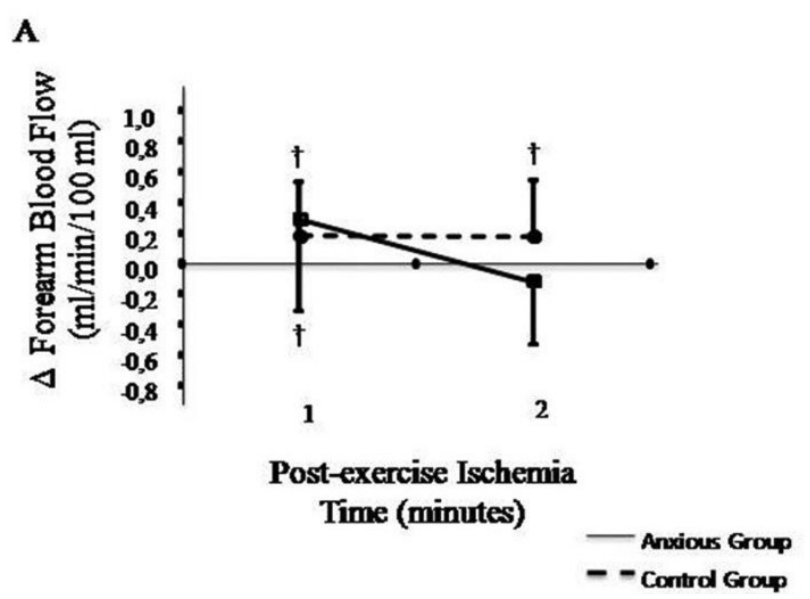

anxious group presented increased forearm vascular resistance. In addition, the increased forearm vascular resistance in the anxious group made this variable higher in this group than in the control group (Figure 4; panel B).

B

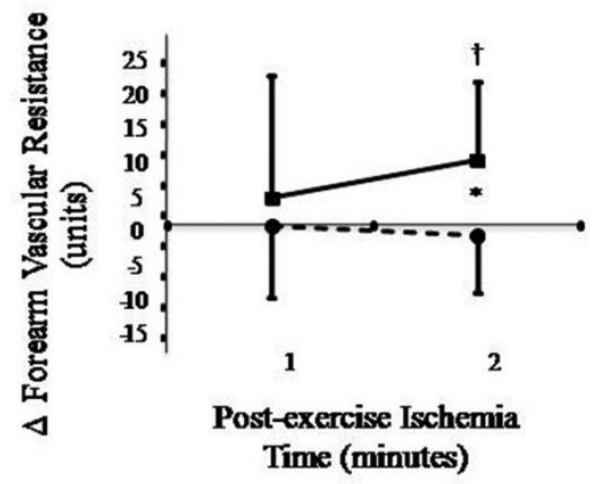

Figure 4. Absolute delta values of the forearm blood flow $(\mathrm{ml} / \mathrm{min} / 100 \mathrm{ml})$ and forearm vascular resistance (units) responses during the postexercise ischemia protocol. Note that muscle blood flow increased similarly in both groups (panel A), whereas forearm vascular resistance increased in both groups when compared with the resting condition, but the anxious group presented more pronounced increases compared with the control group (panel B). Ip $\leq 0.05$ vs. baseline; ${ }^{*} \mathrm{p} \leq 0.05$ vs. group.

Positive and significant correlations were observed between anxiety levels and systolic, diastolic, and mean blood pressures during the rest (Figure 5; panel A, B and $\mathrm{C}$, respectively) and exercise (Figure 6; panel A, B and C, respectively) periods. There were no correlations between anxiety level and heart rate either at rest (Figure 5; panel D) or during exercise (Figure 6; panel D). Additionally, there were no correlations between anxiety level and muscle blood flow at rest $(r=-0.05$ and $\mathrm{p}=0.76)$ and during exercise $(\mathrm{r}=-0.12$ and $\mathrm{p}=0.43$ ) or between anxiety level and forearm vascular resistance at rest $(\mathrm{r}=0.13$ and $\mathrm{p}=0.40)$ or during exercise $(\mathrm{r}=0.24$ and $\mathrm{p}=0.13)$. 
$\mathbf{A}$

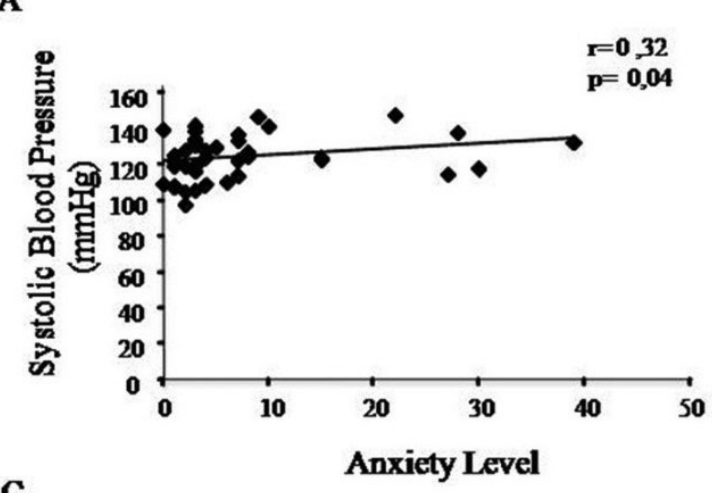

$\mathbf{C}$

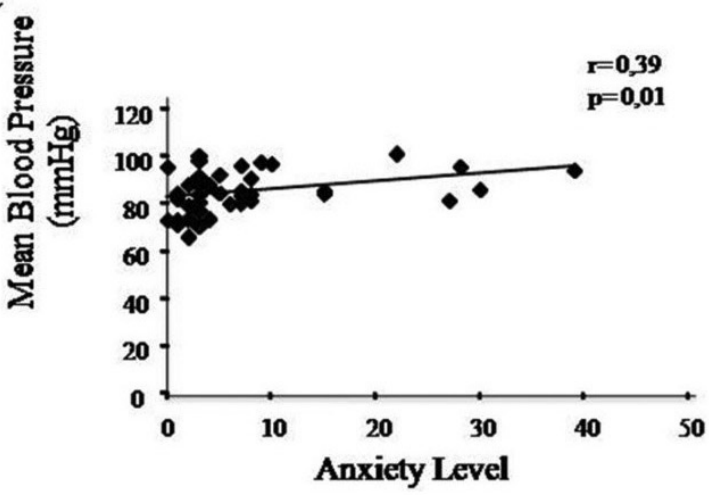

B

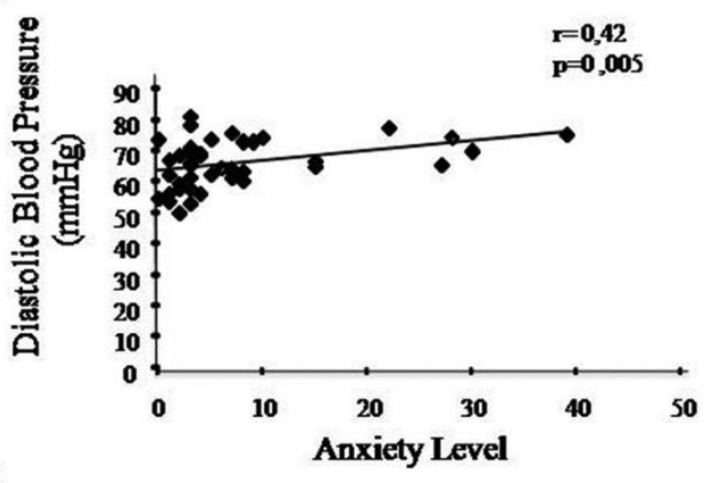

D

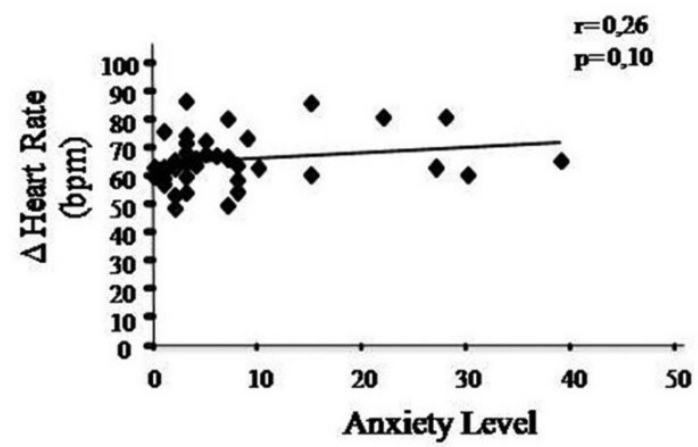

Figure 5. Relationships between anxiety level and systolic, diastolic, and mean blood pressure and heart rate levels (panels A, B, C, and D, respectively) during the rest period. Note that the anxiety level showed directly proportional correlations to systolic, diastolic, and mean blood pressure levels. We did not observe a correlation between anxiety level and heart rate.

A

C

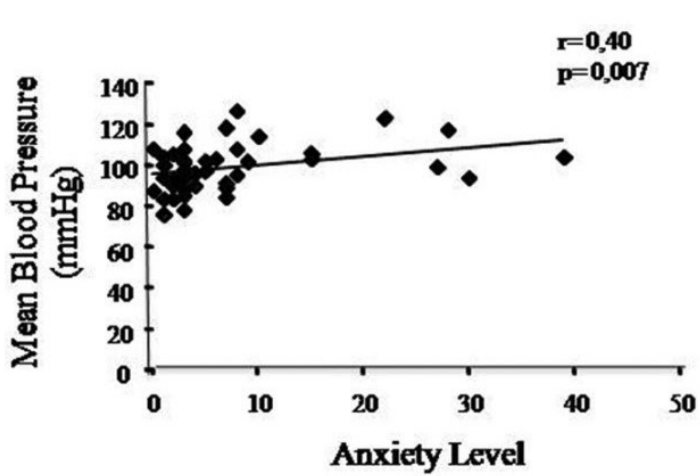

B

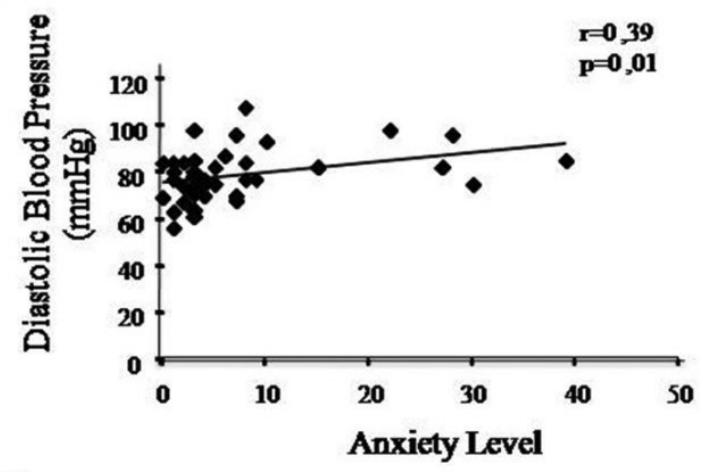

D

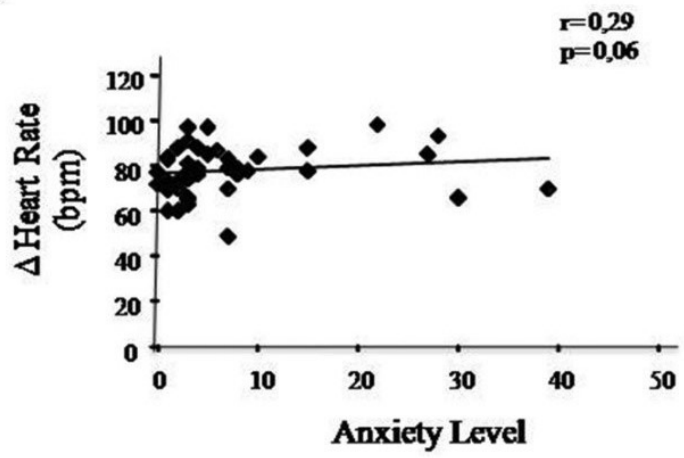

Figure 6. Relationships between anxiety level and systolic, diastolic, and mean blood pressure and heart rate levels (panels A, B, C, and D, respectively) during the handgrip exercise protocol at $30 \%$ of maximal voluntary contraction. Note that the anxiety level showed directly proportional correlations with systolic, diastolic, and mean blood pressure levels. We did not observe a correlation between anxiety level and heart rate. 


\section{Discussion}

The main findings of the present study are those anxious individuals present, in addition to higher resting blood pressure levels, greater reflex pressor of the exercise (greater metaborreflex response) when compared with individuals with minimal anxiety symptoms. Additionally, another important finding was the positive relationship between anxiety level and blood pressure both at rest and during exercise.

Studies have shown that anxious individuals present autonomic changes related to sympathetic hyperactivation ${ }^{19,20}$. Thus, anxiety has been associated with a heightened sympathoadrenal activity, suggesting a biological pathway through which anxiety could increase cardiovascular disease risk $^{19}$.

According to Scalco, Scalco, Azul, Lotufo Neto ${ }^{20}$, hypertensive individuals tend to be hyper-responsive to acute stressors in terms of both blood pressure and increases in total peripheral resistance, suggesting that blood pressure is responsive to the psychosocial environment ${ }^{20}$. Corroborating this finding, in the present study, during the handgrip exercise, the anxious group presented significantly higher increases in systolic, diastolic and mean blood pressure compared with a group with minimal anxiety symptoms. In fact, these results, suggesting that individuals with higher levels of anxiety present greater risks of exacerbated blood pressure response to exercise, thus contributing to a higher risk of developing acute cardiovascular and cerebrovascular events. According to Palmer et $\mathrm{al}^{21}$, the risk for stroke increases progressively with incremental increases in blood pressure above $115 / 75 \mathrm{mmHg}$. Only $1 \mathrm{mmHg}$ increase in systolic blood pressure increases stroke mortality by $2 \%{ }^{21}$. According to this, patients with anxiety and/or depression represent a particularly vulnerable cohort, as they are at raised risk for developing hypertension ${ }^{22}$.

The mechanisms involved in the exacerbate response of blood pressure during physical exercise in anxious individuals are out of the scope of the present study. However, several mechanisms could be hypothesized. In the face of threatening situations, several cerebral regions (e.g. hypothalamus, amygdala, hippocampus, and medial prefrontal cortex) functions as components of an integrated network involved regulating autonomic and behavioral responses to stress ${ }^{23,24,25}$. Beyond to hypothalamic nuclei, it has been documented that the amygdala is essential for the induction and integration of this autonomic response associated with anxiety and fear ${ }^{26,27}$.

Experimental studies reveal that the stimulation of the central nucleus of the amygdala induces a blood pressure response similar to those elicited by activation of cardiovascular bulbar areas ${ }^{28,29}$. We know that the central nucleus of the amygdala sends projections to various structures that are involved in the modulation of arterial blood pressure (i.e. ventrolateral medulla). Curiously, amygdala hyperactivity is a hallmark in the neurobiology of several anxiety disorders (e.g. social anxiety disorder ${ }^{30,31}$ and generalized anxiety disorder $\left.{ }^{31}\right)$.

Thereby, based on these findings, we can speculate that these neural projections between the central nucleus of the amygdala and the ventrolateral medulla may be the mechanism involved in the exacerbated blood pressure response during exercise in anxious individuals, but further studies are needed to confirm this hypothesis.

Even though exercise has been shown to reduce symptoms associated with anxiety disorders and has the potential to lessen the dependability on psychopharmacology ${ }^{32}$, it is known that during isometric exercise, there is a concern that blood pressure may occasionally increase too much, which may increase an individual's risk for an acute cardiovascular or cerebrovascular event ${ }^{12}$. Choi et $\mathrm{al}^{33}$ conducted a study with prehypertensive individuals and observed the hemodynamic mechanisms behind the exaggerated pressor response during handgrip exercise, observing that the increases in mean blood pressure and total peripheral resistance relative to the resting condition were more pronounced in the prehypertensive group than in the normotensive population, suggesting that metaborreflex sensitivity contributes primarily to the increase of these variables in this group ${ }^{33}$. Nevertheless, they presented exacerbated hemodynamic adaptations at rest and during exercise compared to the control group.

During isometric exercise occur a large increase in mean arterial pressure, but only a small increase in cardiac output and this response is probably because systemic metabolic vasodilation is not expressed in isometric exercise, and this mechanism involves, in part, the autonomic nervous system $^{34}$. In the present study, in addition to the anxious group presenting higher levels of resting mean blood pressure and in response to exercise, during post-exercise ischemia, which portrays the metaborreflex response to exercise, the group with higher levels of anxiety had significantly higher levels of mean blood pressure and greater forearm vascular resistance compared to the group with minimal anxiety symptoms. Which shows that anxious individuals probably present an exaggerated blood pressure response to exercise explained in part by the higher metaborreflex response to this condition. Similar results were observed by Delaney ${ }^{35}$ in a similar study, in which it was observed that hypertensive individuals present an exacerbated metaborreflex response, explained by the exaggerated increase mean blood pressure during handgrip exercise ${ }^{35}$.

Although we could not control the health conditions of all volunteers during the day (for example, substance use, daily salt intake), since the anxious group had higher levels of anxiety at the time of the exercise test, this would be considered as an additional reason for increased blood pressure response during exercise and may expose these individuals to a risk that could be detected by observing anxious behaviour during training or with the help of a mental health professional (e.g., psychologist) to obtain an accurate assessment and, if necessary, develop a management strategy for stress and anxiety during exercise. Thus, anxious individuals require greater care regarding the prescription of the exercise to be performed and in the monitoring of the exercise intensity.

The non-assessment of the subjective perception of effort after exercise may be a possible limitation of the present study. Even when performing exercises at similar intensity levels between groups ( $30 \%$ of maximal voluntary contraction), 
we don't know whether anxious individuals respond with a higher subjective perception of effort. Thus, future studies should evaluate the subjective perception of effort in anxious individuals and should also attempt to explain the mechanism between elevated anxiety levels and increased metaborreflex sensitivity. Therefore, we conclude that anxious individuals present higher blood pressure levels at rest and higher blood pressure responses during exercise compared with those with minimal anxiety symptoms. This pronounced increase in the blood pressure response to the exercise presented by the anxious group is compatible with an exaggerated activity of the exercise pressor reflex, mediated in this study, by the greater metaborreflex response in this group. Furthermore, we found a positive correlation between levels of anxiety and systolic, diastolic and mean blood pressure, both at rest and in exercise.

\section{References}

1. Mah L, Szabuniewicz C, Fiocco AJ. Can anxiety damage the brain?. Curr Opin Psychiatry. 2016;29(1):56-63.

2. American Psychiatric Association. Diagnostic and Statistical Manual of Mental Disorders (DSM-5).1980.

3. Schunck T, Erb G, Mathis A, Jacob N, Gilles C, Namer IJ, et al. Test-retest reliability of a functional MRI anticipatory anxiety paradigm in healthy volunteers. J. Magn. Reson. Imaging. 2008; 27 (3): 459-468.

4. Lipman RD, Grossman P, Bridges SE, Hamner JW, Taylor JA. Mental stress response, arterial stiffness, and baroreflex sensitivity in healthy aging. J. Gerontol. A. Biol. Sci. Med. Sci.2002; 57(7): B279-284.

5. Bhui K, Fletcher A. Common mood and anxiety states: gender differences in the protective effect of physical activity. Soc. Psychiatry Psychiatr. Epidemiol. 2000; 35 (1): 28-35.

6. Sale C, Guppy A, El-Sayed M. Individual differences, exercise and leisure activity in predicting affective well-being in young adults. Ergonomics. 2000; 43 (10): 1689-1697.

7. Araújo KCM, Deus LA, Rodrigues FB, Bezerra ME, Sales MM, Rosa TDS, et al. Resistence exercise improves anxiety and depression in middle- age women. J. Phys. Educ.2017; 28.

8. Iellamo F. Neural mechanisms of cardiovascular regulation during exercise. Auton. Neurosci.2001; 90 (1-2): 66-75.

9. Mark A L, Victor RG, Nerhed C, Wallin BG. Microneurographic studies of the mechanisms of sympathetic nerve responses to static exercise in humans. Circ. Res.1985; 57(3): 461-469.

10. Shepherd JT. Circulatory response to exercise in health. Circulation. 1987; 76: VI3-10.

11. Victor R G, Pryor S L, Secher N H, Mitchell J H. Effects of partial neuromuscular blockade on sympathetic nerve responses to static exercise in humans. Circ. Res.1989; 65(2): 468-476.

12. Hoberg E, Schuler G, Kunze B, Obermoser AL, Hauer K, Mautner HP. et al. Silent myocardial ischemia as a potential link between lack of premonitoring symptoms and increased risk of cardiac arrest during physical stress. Am. J. Cardiol.1990; 65 (9): 583-589.
13. Mittleman MA, Maclure M, Tofler GH, Sherwood JB, Goldberg RJ, Muller JE. Triggering of acute myocardial infarction by heavy physical exertion. Protection against triggering by regular exertion. Determinants of Myocardial Infarction Onset Study Investigators. N. Engl. J. Med. 1993; 329 (23): 1677-1683.

14. Goodman J, Thomas S, Burr JF. Physical activity series: cardiovascular risks of physical activity in apparently healthy individuals: risk evaluation for exercise clearance and prescription. Can Fam Physician. 2013;59(1):46-9, e6-e10.

15. Beck AT, Epstein N, Brown G, Steer RA. An inventory for measuring clinical anxiety: Psychometric properties. J. Consult. Clin. Psychol. 1988; 56 (6), 893-897.

16. American College of Sports Medicine. Diretrizes de ACSM para os testes de esforço e sua prescrição. Guanabara Koogan, 2007.

17. Martinez DG, Nicolau JC, Lage RL, Trombetta IC, de Matos LD, Laterza MC, et al. Abnormal muscle vascular responses during exercise in myocardial infarction patients. Int. J. Cardiol.2013; 165(1): 210-212.

18. Paula-Ribeiro M, Garcia MMN, Martinez DG, Lima JRP, Laterza MC. Increased peripheral vascular resistance in male patients with traumatic lower limb amputation: one piece of the cardiovascular risk puzzle. Blood Press. Monit.2015; 20 (6): 341-345.

19. Paine NJ, Watkins LL, Blumenthal JA, Kuhn CM, Sherwood A. Association of Depressive and Anxiety Symptoms With 24-Hour Urinary Catecholamines in Individuals With Untreated High Blood Pressure. Psychosom. Med.2015; 77 (2): 136-144.

20. Scalco AZ, Scalco MZ, Azul JBS, Lotufo Neto F. Hypertension and depression. Clinics. 2005; 60 (3): 241-250.

21. Palmer AJ, Bulpitt CJ, Fletcher AE, Beevers DG, Coles EC, Ledingham JG, et al. Relation between blood pressure and stroke mortality. Hypertension . 1992; 20 (5): 601-605.

22. Liu M-Y, Li N, Li WA, Khan H. Association between psychosocial stress and hypertension: a systematic review and meta-analysis. Neurol. Res. 2017; 39(6): 573-580.

23. Indovina I, Robbins TW, Núñez-Elizalde AO, Dunn BD, Bishop SJ. Fear-Conditioning Mechanisms Associated with Trait Vulnerability to Anxiety in Humans. Neuron. 2011; 69(3): 563-571.

24. Bishop SJ. Neurocognitive mechanisms of anxiety: an integrative account. Trends Cogn. Sci. 2007; 11(7): 307-316.

25. Rauch SL, Shin LM, Wright CI. Neuroimaging Studies of Amygdala Function in Anxiety Disorders. Ann. N. Y. Acad. Sci.2006; 985(1): 389-410.

26. LeDoux J. Fear and the brain: where have we been, and where are we going? Biol. Psychiatry . 1998; 44(12): 1229-1238 .

27. Davis M. The Role of the Amygdala in Fear and Anxiety. Annu. Rev. Neurosci. 1992; 15(1): 353-375.

28. Gelsema AJ, McKitrick DJ, Calaresu FR. Cardiovascular responses to chemical and electrical stimulation of amygdala in rats. Am. J. Physiol.Regul. Integr. Comp. Physiol.1987; 253(5): R712-R718.

29. Iwata J, Chida K, LeDoux JE. Cardiovascular responses elicited by stimulation of neurons in the central amygdaloid nucleus in awake but not anesthetized rats resemble conditioned emotional responses. Brain Res.1987; 418(1): 183-188.

30. Klumpp H, Fitzgerald JM. Neuroimaging Predictors and Mechanisms of Treatment Response in Social Anxiety Disorder: an Overview of the Amygdala. Curr. Psychiatry Rep.2018; 20(10):89. 
31. Hilbert K, Lueken U, Beesdo-Baum K. Neural structures, functioning and connectivity in Generalized Anxiety Disorder and interaction with neuroendocrine systems: A systematic review. J. Affect. Disord.2014; 158:114-126.

32. Carek PJ, Laibstain SE, Carek SM. Exercise for the Treatment of Depression and Anxiety. Int. J. Psychiatry Med.2011; 41(1): 15-28.

33. Choi HM, Stebbins CL, Lee OT, Nho H, Lee JH, Chun JM, et al. Augmentation of the exercise pressor reflex in prehypertension: roles of the muscle metaboreflex and mechanoreflex. Appl. Physiol. Nutr. Metab. 2013; 38(2): 209-215.

34. Kaufman MP, Hayes SG. The Exercise Pressor Reflex. Clin. Auton. Res.2002; 12(6): 429-439.

35. Delaney EP, Greaney JL, Edwards DG, Rose WC, Fadel PJ, Farquhar WB. Exaggerated sympathetic and pressor responses to handgrip exercise in older hypertensive humans: role of the muscle metaboreflex. Am. J. Physiol.Heart Circ. Physiol.2010; 299(5): H1318-H1327.

\section{Acknowledgments}

The authors would like to thank the support of FADEPE Foundation. This study was financed in part by the Coordenação de Aperfeiçoamento de Pessoal de Nível Superior - Brasil (CAPES) - Finance Code 001.

\section{Corresponding author}

Daniel Godoy Martinez

Universidade Federal de Juiz de Fora, Rua José Lourenço Kelmer, s/n, Juiz de Fora, MG, Brasil

Email: danielgmartinez@yahoo.com.br

Manuscript received on July 22, 2018

Manuscript accepted on June 18, 2019

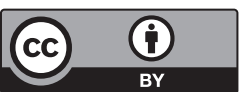

Motriz. The Journal of Physical Education. UNESP. Rio Claro, SP, Brazil - eISSN: 1980-6574 - under a license Creative Commons - Version 4.0 